\title{
Effects of Habenular Stimulation Frequencies on Obstructive Sleep Apnea Induced by Stimulation of Insular Cortex
}

\author{
Jing Kang ${ }^{1,2}$ and Ming-Xian $\mathrm{Li}^{1}{ }^{1}$ \\ ${ }^{1}$ The First Affiliated Hospital of Jilin University, Changchun 130021, China \\ ${ }^{2}$ Jilin Medical University, Jilin 132013, China \\ Correspondence should be addressed to Ming-Xian Li; limingxian_118@163.com
}

Received 31 August 2017; Revised 26 December 2017; Accepted 22 January 2018; Published 26 March 2018

Academic Editor: Emmanuel Charbonney

Copyright ( 2018 Jing Kang and Ming-Xian Li. This is an open access article distributed under the Creative Commons Attribution License, which permits unrestricted use, distribution, and reproduction in any medium, provided the original work is properly cited.

\begin{abstract}
Objective. To investigate the effects of high-frequency stimulation of the habenula $(\mathrm{Hb})$ on obstructive sleep apnea (OSA) induced by stimulation of the insular cortex Method. After OSA was induced by stimulating the insular cortex (Ic) with concentric stimulating electrodes at $100 \mathrm{~Hz}$ in rats, the $\mathrm{Hb}$ was stimulated at different frequencies $(50 \mathrm{~Hz}, 120 \mathrm{~Hz}, 130 \mathrm{~Hz}$, and $280 \mathrm{~Hz})$. The changes of apnea events and electromyography (EMG) of the genioglossus were compared before and after stimulation of the $\mathrm{Hb}$. Results. With stimulation of the Ic at $100 \mathrm{~Hz}$, apnea events were successfully induced with disappearance of EMG of the genioglossus. After stimulation of the $\mathrm{Hb}$ at $130 \mathrm{~Hz}$, apnea events disappeared with significantly increased genioglossal EMG. However, such a change failed to be found at the stimulation frequencies of $50 \mathrm{~Hz}, 120 \mathrm{~Hz}$, and $280 \mathrm{~Hz}$. Conclusion. Stimulation of the $\mathrm{Hb}$ at the frequency of $130 \mathrm{~Hz}$ could effectively inhibit OSA events induced by stimulation of the Ic.
\end{abstract}

\section{Introduction}

Abnormal sleep position, alcohol consumption, drug intake, and obesity can induce obstructive sleep apnea (OSA) [1-3]. Such a complex and highly individualized disease has been associated with multiple cardiovascular diseases. OSA induces chronic hypoxia and hypoventilation that may cause pulmonary hypertension and pulmonary heart disease [4-7]. The predominant pathological causes of OSA include contractility of upper airway muscles, abnormal airway anatomical features, loop gain, and arousal threshold [8]. Since the genioglossus tongue muscle contraction stabilizes and enlarges the portion of the upper airway which is vulnerable to collapse, relaxation of the genioglossus has been implicated in OSA.

According to electroencephalography patterns, which can be used to confirm different stages of sleep, there is a higher likelihood for muscles around the upper airway to relax during deep sleep, thus increasing the possibility of OSA to occur [9]. Previous studies also demonstrated that the electromyography (EMG) activity of the genioglossus is reduced during deep sleep [9]. Subsequently, systolic function decreases with muscle relaxation, which might cause the pharyngeal wall to collapse [10]. Hence, stenosis or closure of the upper airway is usually associated with reduced ventilation or OSA [11-13]. Although recent advancement in neurophysiology has been made in understanding the role of neuronal activity in respiratory movement regulation [13], the pathways regulating the motor control of respiration is not fully explored, especially those regulating the genioglossus contraction, and could be involved in OSA pathogenesis.

Respiratory movement originates from the brainstem and could be regulated by chemoreceptors and mechanoreceptors [12]. In addition, the respiratory movement can also be influenced by signals from the upper portion of the pons and the cerebral cortex [6]. Functional magnetic resonance imaging (fMRI) and animal studies have implicated that the insula cortex plays a major role in the provoking of OSA [7, 8]. In addition, the insular cortex could cause cardiovascular system dysfunction, which is consistent in OSA patients [9]. On the other hand, previous studies have shown that the $\mathrm{Hb}$ can mediate the neuronal signaling from the insula cortex. Inhibition of the $\mathrm{Hb}$ was able to abrogate 
OSA induced by electrical stimulation of the insula cortex [10]. However, it is unclear whether the amplitude of the frequency used to stimulate the $\mathrm{Hb}$ is critical to achieve the maximum response in OSA. In this study, we investigated the effect of different $\mathrm{Hb}$ stimulation frequencies on obstructive sleep apnea. The breathing pattern as well as the EMG activities of the genioglossus was recorded before and after OSA was induced.

\section{Materials and Methods}

2.1. Animal Preparation. This study was carried out in accordance with the Guide for the Care and Use of Laboratory Animals of the National Institutes of Health. The protocol was approved by the Animal Care and Use Committee of the Jilin University (Permit number: SCXK, Kyrgyzstan, 2007-0003, and SYXK, Kyrgyzstan, 20070011). Wistar rats (200-280 g, 6-8 weeks old, $N=92)$ were obtained from the Jilin University Norman Bethune Medical Department Laboratory Animal Center. The animals were housed under a 12/12-hour light/dark cycle in a temperature-controlled room (maintained at $22 \pm 2^{\circ} \mathrm{C}$ ) with ad libitum access to food and water [11]. All surgical and animal handling procedures were performed in accordance with Guidance Suggestions for the Care and Use of Laboratory Animals issued by the Ministry of Science and Technology of the People's Republic of China. The animals were randomly divided into three groups, the OSA group $(n=24)$, Hb group $(n=48)$, and control group $(n=20)$, using SPSS software. For the OSA group, the insula cortex was stimulated to induce OSA. For the $\mathrm{Hb}$ group, the insula cortex and $\mathrm{Hb}$ were stimulated with four different frequencies ( $n=12$ for each frequency). For the control group, the surrounding tissue of either the insula cortex $(n=10)$ or the $\mathrm{Hb}(n=10)$ was stimulated. The breathing pattern and the EMG activities of the genioglossus and diaphragm were recorded before and after stimulation.

All experimental procedures were performed under anesthesia via intraperitoneal injection of $20 \%$ urethane $(6 \mathrm{~mL} / \mathrm{kg})$, and all efforts were made to minimize suffering. During the surgery, the animals were breathing steadily and maintained in a state of light anesthesia (sensitive corneal reflex and forceps pinch produced withdrawal but no sustained response and no voluntary activities) [14] throughout the experimental procedure. Anesthesia was supplemented if needed during the experiment. The animals were maintained at $36^{\circ} \mathrm{C}$ rectal temperature with a heating pad. During the experiment, the animals were fixed under the stereotaxic instruments (Takahashi Company, Japan) with blunt ear bars, and a small craniotomy was performed. For the OSA group, an electrode was implanted into the insula cortex (anterior/posterior: $1.0 \mathrm{~mm}$; medial/lateral: 5.4-5.5 mm; and dorsal/ventral: $4.2-4.4 \mathrm{~mm}$ ), according to the rat brain atlas [15]. Whereas in the $\mathrm{Hb}$ group, electrodes were implanted into the insula cortex (Ic) and $\mathrm{Hb}$ (anterior/posterior: $-3.2 \mathrm{~mm}$; medial/lateral: $0.3-0.6 \mathrm{~mm}$; and dorsal/ventral: $3.8-4.2 \mathrm{~mm}$ ). The control group received electrical stimulation with the same parameters, but the electrode locations were at four points (top, bottom, left, and right) within a $1 \mathrm{~mm}^{2}$ range around the Ic. The rats were fixed in a ventral decubitus position and breathed spontaneously during the experiments.

\subsection{Electrical Stimulation and Recording Protocols. Electrical} stimulation device (Nihon Kohden, Japan) and concentric stimulating electrodes (Nihon Kohden, Japan; outer diameter of $2.0 \mathrm{~mm}$ and impedance of $10-15 \mathrm{M} \Omega$; Figure 1) were used for stimulation. OSA was induced by stimulating the Ic at $100 \mathrm{~Hz}, 0.4 \mathrm{~mA}$, and $1 \mathrm{~ms}$ interval for $9 \mathrm{~s}$ [16]. Signs of apnea and respiratory distresses were used as indicators of successful induction during the experiment. Histology analysis of induction site was also performed. No signs of apnea and respiratory distress were observed in the control group. In the $\mathrm{Hb}$ group, the $\mathrm{Hb}$ was stimulated continuously for at least $30 \mathrm{~min}$ at $50 \mathrm{~Hz}, 120 \mathrm{~Hz}, 130 \mathrm{~Hz}$, or $280 \mathrm{~Hz}$ before and after the insula cortex was stimulated.

The breathing pattern and EMG were recorded using a data logger (BL-420S, Chengdu Taimeng Technology Co., Ltd.). A respiratory flow transducer and a tension transducer were utilized to monitor the respiratory flow via endotracheal intubation and respiratory rates, respectively. The lead electrode was inserted to the genioglossus and diaphragm to record the EMG signals in the genioglossus and diaphragm, respectively.

2.3. Data Analysis. The electromyography (EMG) of the genioglossus and diaphragm, as well as the respiratory amplitude and respiratory rate, was compared before and after the stimulation of the Hb. SPSS 19.0 was used for all statistical analysis. Data were presented as mean \pm standard deviation $(X \pm S) . P<0.05$ was considered as statistically significant.

\section{Results}

Concentric stimulation of the Ic was performed in 12 OSA rats. During stimulation, weakened genioglossus contraction, apnea, interruption of airflow, and reduction of the respiratory rate were observed in OSA rats. Whereas, after stimulation, signs including intermittent apnea, a significant reduction of airflow (from $89.08 \pm 4.21$ breaths $/ \mathrm{min}$ to $12.19 \pm 8.49$ breaths/min), transbreathing, reduction in the respiratory rate, deep breathing after stimulation, airflow return to baseline in few seconds, and significant reduction of genioglossus discharge were observed (Figure 2). Subsequently, the $\mathrm{Hb}$ was stimulated continuously for $30 \mathrm{~min}$ at $50 \mathrm{~Hz}, 120 \mathrm{~Hz}, 130 \mathrm{~Hz}$, and $280 \mathrm{~Hz}$ in the same rat. Simultaneous concentric stimulation of the Ic was performed using the same parameters, and the breath and EMG activities of the genioglossus were monitored. As shown in Figure 3, the respiration rate and amplitude as well as EMG patterns of the genioglossus were returned within normal ranges after $130 \mathrm{~Hz}$ stimulation. Notably, the respiration rate changes at different stimulation frequencies of the $\mathrm{Hb}$ in OSA-induced animal models (Figure 4). 


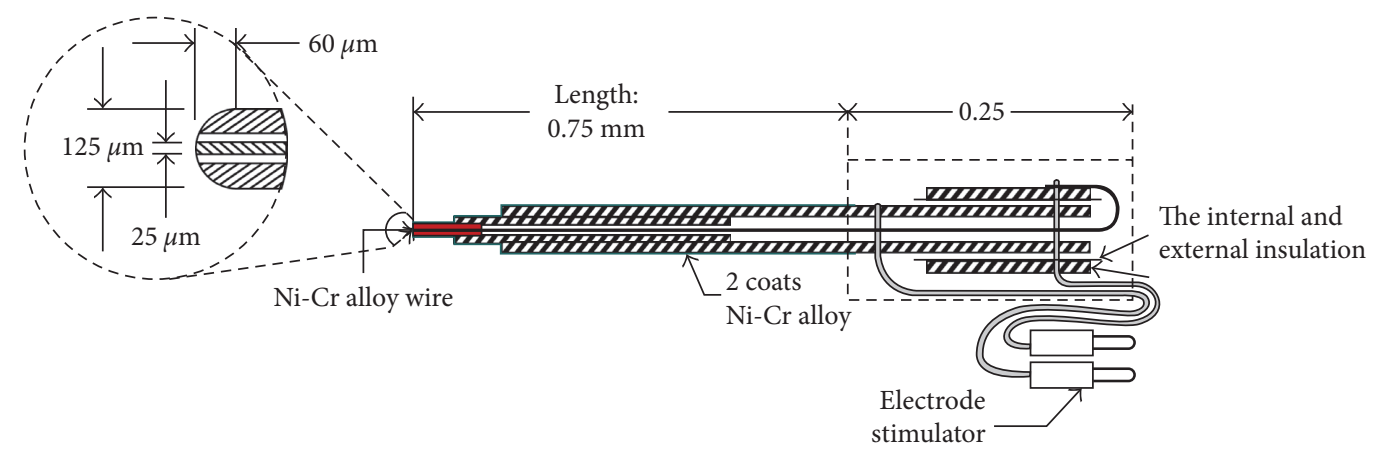

Figure 1: Schematic of stimulation electrode. The experimental stimulation electrode is a Ni-Cr alloy wire concentric exciter electrode of Japanese Narishige Company.
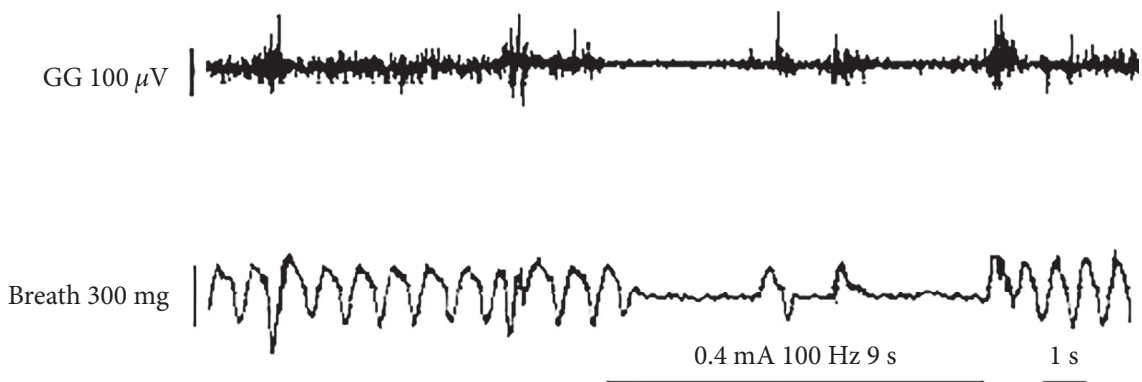

FIgUre 2: Typical changes in respiration and genioglossus EMG patterns before, during, and after stimulation in the IC with $100 \mathrm{~Hz}$.

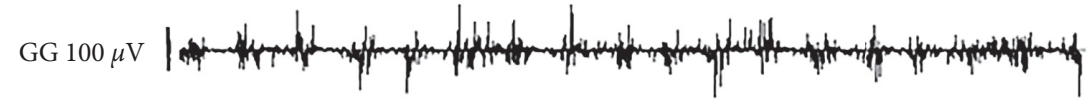

Breath $300 \mathrm{mg}$

$\stackrel{0.4 \mathrm{~mA} 130 \mathrm{~Hz} 30 \mathrm{~min}}{1}$

Figure 3: Typical respiration and genioglossus EMG patterns after $130 \mathrm{~Hz}$ stimulation of the $\mathrm{Hb}$ in the OSA-induced animal.

\section{Discussion}

The genioglossus has been commonly considered as the pathophysiology of OSA. Horner et al. reported that the genioglossus contractile function was significantly decreased during sleep [17]. Our results showed a reduction in genioglossus contraction in OSA rats, which is consistent with a previous study showing that genioglossus activity significantly reduced during sleep in OSA patients with subsequent weakening in contraction resulting in muscle relaxation in the pharyngeal wall [18]. As a consequence, a reduction in the respiratory rate was observed (Figure 2), which may be attributed to the subsequent collapse of the upper airway, thus reducing ventilation [18]. It should be noted that, despite the application of excitatory neurotransmitter serotonin (5-hydroxytryptamine, 5-HT), sleep could not prevent genioglossus diastole [19]. Thus, the main reason for the occurrence of OSA is associated with respiratory disorders, suggesting that the genioglossus could play an important role in OSA. The genioglossus electrical activity weakens during sleep, causing the muscles to relax and a reduction in ventilation. Consequently, chronic intermittent hypoxia or hypercapnia (chronic intermittent hypopnea or hypercapnia, $\mathrm{CIHH}$ ) occurs, or even acidosis.

Our results showed that $100 \mathrm{~Hz}$ concentric stimulation of the Ic could induce OSA in rats. The Ic, a region of the limbic system, can change the genioglossus tension, as well as the breathing depth and rate. Many neurotransmitters regulating the limbic system are associated with many diseases [20-23]. Signals propagating from the Ic to the $\mathrm{Hb}$ could reduce the release of $5-\mathrm{HT}$ in the raphe nuclei. Subsequently, the activity of hypoglossal nucleus would decrease, resulting in the reduction of genioglossus contractile function. Consequently, genioglossus relaxation occurs, which can cause airway to collapse, and thus OSA. It should be mentioned that the Ic is involved in regulating OSA and has been related to respiratory motion adjustments [11]. The activities in the Ic increased during OSA, and inhibition could occur in the raphe nucleus that reduces the release of 5-HT. Subsequently, the activity of the hypoglossal 


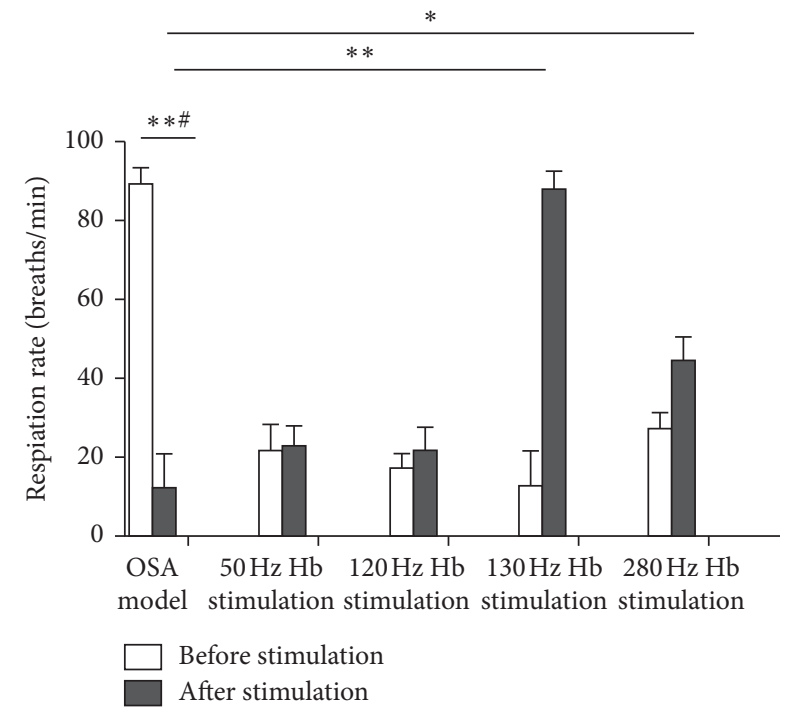

FIgURE 4: Respiration rate changes at different stimulation frequencies of the $\mathrm{Hb}$ in OSA-induced animal models. $* * \#$ denotes $P<0.001$ before and after stimulation in the insula cortex, while ** and $*$ denote $P<0.001$ and $P<0.05$, respectively, between the OSA model and different stimulation frequencies in the habenula.

nucleus and the hypoglossal nerve excitability are reduced. Thus, the genioglossus contraction is weakened, and the upper airway will be more vulnerable to collapse causing OSA.

When the $\mathrm{Hb}$ was stimulated continuously for $30 \mathrm{~min}$ at $130 \mathrm{~Hz}$, the EMG patterns of the genioglossus returned within normal ranges. These results indicate that such a stimulation can be used to treat OSA. The Hb plays an important role in OSA, since the $\mathrm{Hb}$ mediates signals from the Ic to the downstream. Consequently, $130 \mathrm{~Hz}$ stimulation may block the $\mathrm{Hb}$, resulting in genioglossus contraction, and thus, the upper airway may open and ultimately improve ventilation. These results indicate that inhibition of the $\mathrm{Hb}$ can block downstream signal propagation from the Ic, thereby avoiding the formation of OSA. Our results reveal the relationship between $\mathrm{Hb}$, OSA, and Ic and may also provide a new therapeutic strategy in OSA.

\section{Conclusion}

Electrical stimulation of $130 \mathrm{~Hz}$ could inhibit $\mathrm{Hb}$, which reduces downstream signal propagation initiated by the Ic. This, in turn, will cause the genioglossus contractile function to recover, avoiding the formation of obstructive sleep apnea.

\section{Conflicts of Interest}

The authors declare that they have no conflicts of interest.

\section{Acknowledgments}

The authors thank Professor Shao Wang for assistance with the experiments, who passed away 2 years ago; he provided constructive advice to conceive the study. The authors are grateful to all the participators of the Department of Physiology Lab and the Center of Medical Laboratory of Jilin Medical University. The study was supported by the National Natural Science Foundation of China (81670080) and the National College Students Innovation and Entrepreneurship Training Program (201613706024). The sponsor had no role in the design or conduct of this research. The authors thank Sleep Medicine for presenting this work as an abstract in December 2017.

\section{References}

[1] F. G. Issa and C. E. Sullivan, "Alcohol, snoring and sleep apnea," Journal of Neurology, Neurosurgery, and Psychiatry, vol. 45, no. 4, pp. 353-359, 1982.

[2] W. Richard, D. Kox, C. den Herder, M. Laman, H. van Tinteren, and N. de Vries, "The role of sleep position in obstructive sleep apnea syndrome," European Archives of Oto-Rhino-Laryngology, vol. 263, no. 10, pp. 946-950, 2006.

[3] A. Romero-Corral, S. M. Caples, F. Lopez-Jimenez, and V. K. Somers, "Interactions between obesity and obstructive sleep apnea: implications for treatment," Chest, vol. 137, no. 3 , pp. 711-719, 2010.

[4] N. A. Dewan, F. J. Nieto, and V. K. Somers, "Intermittent hypoxemia and OSA: implications for comorbidities," Chest, vol. 147, no. 1, pp. 266-274, 2015.

[5] S. Q. Yang, L. L. Han, X. L. Dong et al., "Mal-effects of obstructive sleep apnea on the heart," Sleep and Breathing, vol. 16, no. 3, pp. 717-722, 2012.

[6] M. Kohler and J. R. Stradling, "OSA and hypertension: do we know all the answers?," Chest, vol. 144, no. 5, pp. 1433-1435, 2013.

[7] M. X. Li, J. H. Wang, M. Huang et al., "Blockage of the habenular nucleus can eliminate dyspnea induced by electrostimulation of the insular cortex," Neural Regeneration Research, vol. 5, no. 13, pp. 1025-1029, 2010.

[8] J. P. Saboisky, N. L. Chamberlin, and A. Malhotra, "Potential therapeutic targets in obstructive sleep apnoea," Expert Opinion on Therapeutic Targets, vol. 13, no. 7, pp. 795-809, 2009.

[9] A. Vakulin, A. D’Rozario, J. W. Kim et al., "Quantitative sleep EEG and polysomnographic predictors of driving simulator performance in obstructive sleep apnea," Clinical Neurophysiology, vol. 127, no. 2, pp. 1428-1435, 2016.

[10] L. K. Huon, S. Y. Liu, T. T. Shih, Y. J. Chen, M. T. Lo, and P. C. Wang, "Dynamic upper airway collapse observed from sleep MRI: BMI-matched severe and mild OSA patients," European Archives of Oto-Rhino-Laryngology, vol. 273, no. 11, pp. 4021-4026, 2016.

[11] L. Cui, J. H. Wang, M. Wang et al., "Injection of L-glutamate into the insular cortex produces sleep apnea and serotonin reduction in rats," Sleep and Breathing, vol. 16, no. 3, pp. 845-853, 2012.

[12] J. Li, M. X. Li, S. N. Liu et al., "Is brain damage really involved in the pathogenesis of obstructive sleep apnea?," Neuroreport, vol. 25, no. 8, pp. 593-595, 2014.

[13] M. X. Li, C. Y. Yan, and S. Wang, "New insights on the role of the insular cortex and habenula in OSA," Sleep and Breathing, vol. 19, no. 4, pp. 1347-1353, 2015.

[14] H. L. Fields, J. Bry, I. Hentall, and G. Zorman, “The activity of neurons in the rostral medulla of the rat during withdrawal from noxious heat," Journal of Neuroscience, vol. 3, no. 12, pp. 2545-2552, 1983. 
[15] P. George and W. Charles, The Rat Brain in Stereotaxic Coordinates, Academic Press, Cambridge, MA, USA, 6th edition, 2006.

[16] S. Wang, Q. Fu, and J. Fu, "Modulation of preoptic area on discharges of parafasc insular pain neurons," Ke Xue Tong Bao, vol. 28, pp. 401-405, 1983.

[17] R. L. Horner, X. Liu, H. Gill, P. Nolan, H. Liu, and S. Sood, "Effects of sleep-wake state on the genioglossus vs. diaphragm muscle response to $\mathrm{CO}(2)$ in rats," Journal of Applied Physiology, vol. 92, no. 2, pp. 878-887, 2002.

[18] S. A. Shea, J. K. Edwards, and D. P. White, "Effect of wakesleep transitions and rapid eye movement sleep on pharyngeal muscle response to negative pressure in humans," Journal of Physiology, vol. 520, no. 3, pp. 897-908, 1999.

[19] K. A. Sillay, D. Rusy, L. Buyan-Dent, N. L. Ninman, and K. K. Vigen, "Wide-bore 1.5 T MRI-guided deep brain stimulation surgery: initial experience and technique comparison," Clinical Neurology and Neurosurgery, vol. 127, pp. 79-85, 2014.

[20] S. Vallabhajosula, I. U. Haq, N. Hwynn et al., "Low-frequency versus high-frequency subthalamic nucleus deep brain stimulation on postural control and gait in Parkinson's disease: a quantitative study," Brain Stimulation, vol. 8, no. 1, pp. 64-75, 2015.

[21] A. M. Lozano, L. Fosdick, M. M. Chakravarty et al., "A phase II study of fornix deep brain stimulation in mild Alzheimer's disease," Journal of Alzheimer's Disease, vol. 54, no. 2, pp. 777-787, 2016.

[22] J. A. Espinoza Martinez, G. J. Arango, E. T. Fonoff et al., “Deep brain stimulation of the globus pallidus internus or ventralis intermedius nucleus of thalamus for Holmes tremor," Neurosurgical Review, vol. 38, no. 4, pp. 753-763, 2015.

[23] S. K. Yadav, R. Kumar, P. M. Macey, M. A. Woo, F. L. Yan-Go, and R. M. Harper, "Insular cortex metabolite changes in obstructive sleep apnea,” Sleep, vol. 37, pp. 951-958, 2014. 